\title{
Basic Problems in Self-Assembling Robots and a Case Study of Segregation on Tribolon Platform
}

\author{
Shuhei Miyashita, Aubery Marchel Tientcheu Ngouabeu, Rudolf M. Füchslin, \\ Kohei Nakajima, Christof Audretsch, and Rolf Pfeifer
}

\begin{abstract}
It has been a quite while since people realized that self-assembly technique may be a strong method to manufacture $3 \mathrm{D}$ micro products. In this contribution, we investigate some major concerns about realizing such a small sized robot. First we introduce the concept of self-assembly and introduce examples both from nature and artificial products. Followed by the main problems in self-assembly which can be seen in various scales, we classify them into four groups - (A) assembly constraint issues, (B) stochastic motion issues, (C) interactions on physical property issues, and (D) engineering issues. Then we show a segregation effect with our developed platform as an example of self-organizing behavior achieved in a distributed manner.
\end{abstract}

\section{Self-assembly}

One of the major features of biological systems is that the activities on the molecular level are realized in a decentralized fashion, namely, without any central control. One aspect of this phenomenon is self-assembly, defined by Whitesides et al. as the autonomous organization of components into patterns or structures without human intervention 37 . Such a new composition method has large potential in manufacturing 3D micro products, where a pick-and-place style fabrication method is still the major approach taken.

Shuhei Miyashita · Aubery Marchel Tientcheu Ngouabeu · Rudolf M. Füchslin ·

Kohei Nakajima $\cdot$ Christof Audretsch · Rolf Pfeifer

Artificial Intelligence Laboratory Zurich,

University of Zurich, Andreasstrasse 15, 8050 Zurich

e-mail:miyalifi.uzh.ch

${ }^{1}$ Note the notion self-assembly does not only contain instance of decentralized functionality.

Y. Meng and Y. Jin (Eds.): Bio-Inspired Self-Organizing Robotic Systems, SCI 355, pp. $173-191$ springerlink.com

(c) Springer-Verlag Berlin Heidelberg 2011 


\subsection{Self-assembly in Nature}

The instances of self-assembly can be widely found in nature - snow flakes, in which the composing atoms form ordered lattices through the attractive/repulsive interaction forces, employs self-assembly for its spontaneous crystallization. The shapes are commonly hexagonal, but the details of the patterns vary depending on the environmental conditions, such as humidity or temperature. The crystallization begins with a "core" - often a spec of dust in the air - allowing other floating atoms to connect to the seed. Once the atoms connect to the crystal, they change their conformations, exposing the other connection sites, allowing further atoms to attach. In other words, information of the connections is conveyed to external atoms. The system is conservative in terms of energy dispersion, that is, once an atom connects to the cluster and changes the form, it preserves the energy and sustains the formation by means of a hydrogen bond, unless the temperature rises and breaks the bond.

Lately, this kind of automatic assembly has been brought to attention as the difficulty of manufacturing small sized robots starts to be a limiting factor. Now many researchers believe it sounds reasonable to consider self-assembly as a promising tool to be put into practice for the realization of life like machines (e.g. self-repairable machines). However, despite nature's efficiency and precision in assembling supramolecular and mesoscopic structures, most of the attempts on artificial self-assembly components still remain a challenging assignment.

\subsection{From Self-assembling Blocks to Self-assembling Robots}

Progressive experiments on artificial self-replication were conducted by Lionel and Roger Penrose half a century ago [27], where a provoking mechanical model of natural self-replication in a stochastic environment was presented. Followed by speculations about the clustering patterns of passive elements, focusing on the role of shape on template and components matching [10], and on their time evolution [18]. A series of studies were conducted by the group of Whitesides; for the realization of positional coordinate of molecule-mimetic chemistry [7, 6, 17, 38], circuit functionality [13, 5, 4], reversible aggregation [22], folding structure [3], rotation of magnets [16], rotation of rotors [15]. Similarly, numerous research effort has been devoted to the investigation of morphology [31]. Artificial chemicals that can form in several ways, such as polymers and dimers, depending on the temperature of the system were demonstrated in [8]. Different aggregation patterns with various sizes of components were shown in [39]. An intelligent self-assembling block which can represent multiple states by the units' rotational angle was designed by [34]. The system can physically conduct XOR calculation on a 2D plane.

Currently, there is a growing interest in realizing self-reconfigurable robots relying on stochastic self-assembly. White et al. studied two systems in which the modules binding preferences are coded in a program executed by an on-board microcontroller, and thus can easily reconfigure the structure [35]. The modules are initially unpowered and passive, but once they bind to a seed module connected to a power supply, they become active. Griffith et al. studied a system of template-replicating 
modules [14]. They used modules of the same type, which are programmable and can store distinct states. The system demonstrated the self-replication of a five modules polymer. Each module executed a finite-state machine. Klavins et al. examined the problems of designing a grammar that causes modules to assemble into desired products, of predicting the time complexity of such processes, and of predicting (and optimizing) the yield of such processes [20]. Emergent self-propulsion mechanisms were investigated by Ishiguro et al. [19]. In Ant-inspired robotics, the interest in self-organization has been driven by the observations of the same phenomena in ant colonies, in particular the brood sorting by Temnothorax [30]. Wilson et al. [21] created an algorithm to realize two colors annular sorting which used differential pull-back distances for different object types. By discriminating between three puck types, the robots could drop the first type of object on colliding with another puck, drop the second object type after pulling back a short distance and drop the third puck type after pulling back a further distance.

\section{Major Concerns in Self-assembly}

In this section, we outline the problems of self-assembling systems with respect to the scaling behavior of underlying principles.

\subsection{The Forward Problem and the Backward Problem}

In self-assembly, the problem to derive the final configuration from a given set of components/environments is called the forward problem [26]2. Conversely, the problem of designing components for a targeted configuration is called the backward problem. In this "reverse engineering" process, also known as one of the central problems in self-assembly, the designer has to start from the final structure and decompose it.

Several aspects of both, the forward and the backward problem, show strong dependence on the length scale of the system. A necessary condition for the prediction of the result of a self-assembly process is a detailed knowledge about the morphology of the components. And this knowledge is easier to get the larger the components are 3 . In contrast to $\mathrm{cm}$-sized components, a molecule usually has many degrees of freedom and therefore the morphology and consequently the interaction between components are not always known with sufficient precision.

Similar considerations hold for the backward problem, and, from an engineering perspective, to an even higher degree. Given an object $O$ for which one wants to design a self-assembly process. There are many possibilities to divide a large $O$ into components. The smaller $O$, the more constraints with respect to the production of

\footnotetext{
2 The game Tetris ${ }^{\circledR}$ is known as NP-hard problem [12]. Also it may be useful to mention that some situations in self-assembly resemble the Knapsack problem, which is also known as NP-complete problem.

3 This does not only refer to shape but also to other features of morphology, such as elasticity and degrees of freedom.
} 
a)

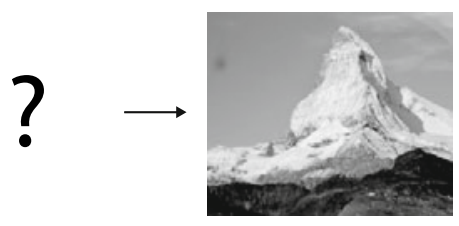

b)

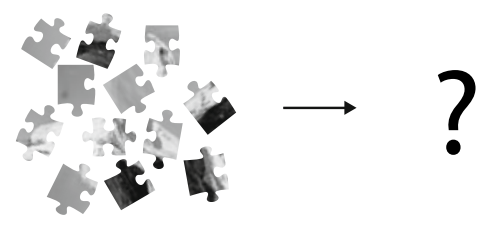

Fig. 1 (a) The backward problem. (b) the forward problem.

the components have to be considered: On the molecular scale, chemical synthesis set narrow limits to what can be accomplished.

We recognize three main problems centering around self-assembly: namely, the issues entailed in (A) assembly, (B) Dynamics, and (C) interactions. The explanations for each problem follow.

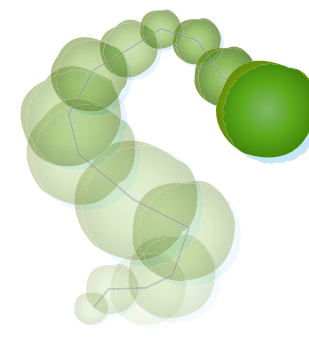

(B) Dynamics issues

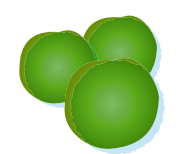

(A) Assembly issues

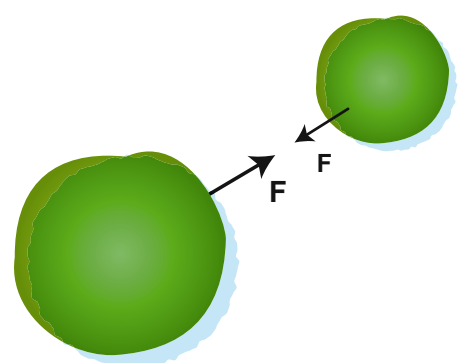

(C) Interaction issues

Fig. 2 Three main issues centering around self-assembly.

\section{2 (A) Assembly}

The first issues are properties of assembling processes.

\subsubsection{The Mismatch Problem (Error)}

In self-assembly, an assembly error (or wrong attachment) is induced when the system converges to a energetically local minimum through interactions between components, mainly due to the low encoding accuracy of bonding sites (Figure 3). 


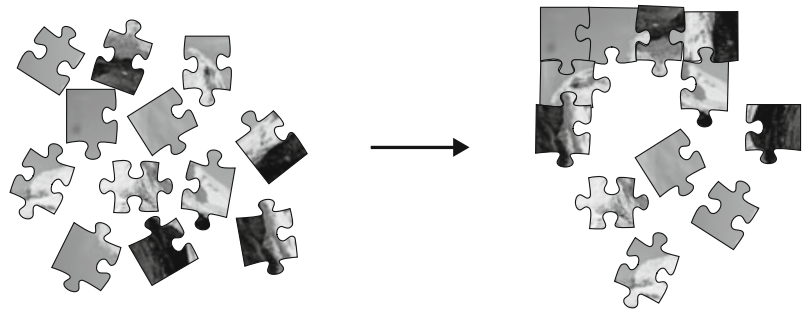

Fig. 3 The mismatch problem (Error).

There are two main strategies for solving the problem; increasing encoding accuracy of bond matching while regulating the agitation level of the system, and implementing internal states to components. Insights from molecular biology remind us of the importance of the fertile encoding capability to attain the adequate bonding affinity level for maintaining connections of molecular bonding; they exploit non-covalent bonds (hydrogen bonds, ionic bonds, and van der Waals attractions) as interaction forces and somehow achieve an amazing specificity in docking with other selected molecules 4 . The agitation level can be regulated by means of temperature or kinetic turbulence magnitudes.

From an engineering perspective, the scaling behavior of the mismatch problem exhibits an interesting feature. The relative easiness of the backward problem enables one to construct highly specific, literal plug-and-socket connection sites on the $\mathrm{cm}$ scale.

Molecules, on the other hand, may well be highly flexible (having many degrees of freedom) and, agitated by thermal motion, "sample" their configuration space at a rapid pace. Because of this, e.g. two complementary DNA strands just have be brought in close proximity: If they match, they will eventually bind. Such a "fast configuration sampling" is not possible above the molecular scale. One of the reasons for this is that whereas mechanical structures wear off, molecules don't: A molecular "joint" can be bended infinitely many times (as long as the bond doesn't break, it is in all respects as good as a newly formed one). It is the micro-to (sub)millimeter scale, at which molecular bonding (and corresponding recognition) is not strong enough anymore, whereas mechanical plug-and-socket connection mechanisms are still hard to produce.

The mismatch problem is a fundamental problem in nature. The replication processes of DNA is greatly assisted by self-repair functionalities of enzymes.

\subsubsection{The Topological Dead End Problem (Steric Hindrance)}

This problem occurs when components assemble in an undesired sequential order. The targeted structure is therefore unreachable, since some earlier assembled components block the way (Figure 4).

\footnotetext{
4 The trick of proteins distributing bonding sites around the body, and changes the morphology to pose another bonding site reminds us of the importance of internal states, which enables the component to feature different properties.
} 


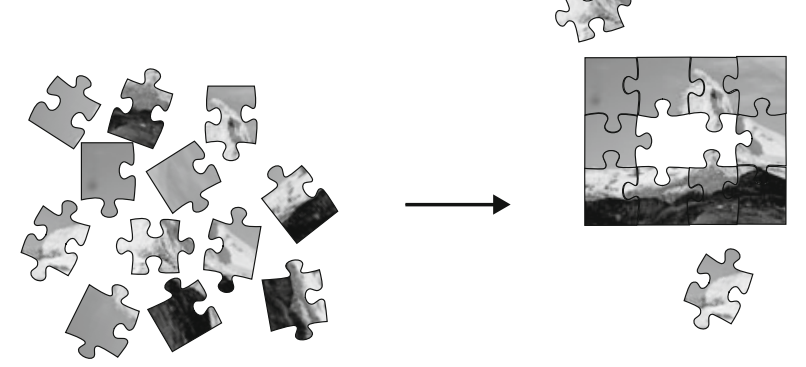

Fig. 4 The topological dead end problem (Steric hindrance).

To solve this problem at scales where the benefits of molecular mechanical flexibility cannot anymore be harvested, the components should reflect the presence of its neighbors e.g. as the internal states. Yet in practice the amount of expressible internal states is limited due to the limited space in a component, leading to a risk of misrecognition by other components.

The unreachable problem can occur irrespective of the heterogeneous/ homogeneous level of a system.

\subsubsection{The Parallel Yield Problem (Incompletion Problem)}

The problem of producing a desired configuration in large quantities (while avoiding incomplete assemblies) by homogeneous system is known as the parallel yield problem and has been studied in the context of biological and non-biological selfassembling systems [18 5. Here, we term the problem that specifically occurs when components assemble in a right manner, however do not complete the targeted final structure, for combinatorial reasons (Figure 5, we assume the circular sector components connect side-by-side). This is because many assembly processes proceed in parallel and components are used in earlier more likely reactions of other assembly processes since reactions leading to the complement of the end product are more unlikely than the preceding reactions. This means that the self-assembly of many products is started but rarely fulfilled. In other words, the likelihood to accomplish the desired end product declines with the rise of the ratio between the likelihood of the earlier and the later reaction.

One approach to improve this problem is, therefore, controlling the system so that the later stages' reactions to be more likely to happen than the early stages. This can be handled by implementing internal states in a component, so that the component behaves differently as a reaction takes place.

Another solution is increasing the heterogeneity of the system, such that in extreme, a product consists of a set of totally different components. Yet a certain

\footnotetext{
${ }^{5}$ Hosokawa called it "yield problem", while we term it the parallel yield problem to avoid confusion.
} 


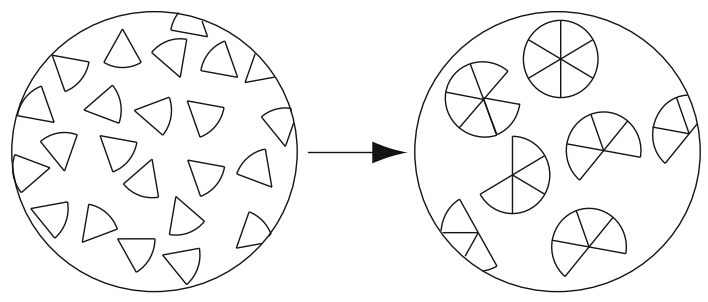

Fig. 5 The parallel yield problem (Incompletion problem).

disadvantage of heterogeneity increase is, as described above, that leads to the heightened likelihood of mismatching.

\section{3 (B) Dynamics}

Self-assembly is commonly believed to range from molecular to cosmological scales. However, it is also agreeable that few examples of self-assembly exist in our human living scales $(\mathrm{cm}-\mathrm{m})$. The second concern is about stochasticity, which varies to different scales.

\subsubsection{Reynolds Number}

Biological systems in the $n m-\mu m$ scale often show unique behaviors that cannot be observed in larger scales. This is mostly due to the influence of viscosity, which increasingly becomes dominant with decreasing length scales. The Reynolds number $\Re$ represents a ratio between viscous forces and inertial forces [28];

$$
\Re \equiv \frac{\text { inertial forces }}{\text { viscous forces }} \approx \frac{a v \rho}{\eta}=\frac{a v}{v} \text {. }
$$

where $a$ is radius of a particle, $v$ is its speed, $\mu$ is fluid viscosity, and $\rho$ is fluid density. The kinematic viscosity $v$ is approx. $10^{-2} \mathrm{~cm}^{2} / \mathrm{sec}$ for water.

The size of $1 \mathrm{~cm}$ is a critical size for self-assembling systems. For objects in water at the $m m$ scale, viscosity is as important as inertia (the Reynolds number, that is, the ratio of inertial forces and viscous forces, is $\approx 1$ ). It follows that objects smaller than that size are affected more by viscous forces whereas larger objects are affected more by inertial forces. For objects on the order of $1 \mu \mathrm{m}$ or less, such as bacteria, exploiting an environmental diffusion is a more effective way of locomotion than active propulsion (e.g., swimming bacteria are slower than diffusing molecules [24]). Good thought-provoking suggestions about the life at low Reynolds number are introduced in [28]. The author states the efficiency of creatures in small scale $(\mu \mathrm{m})$ such as E. coli to use diffusion through their environment to change their position, rather than self-propelling. Whitesides implies the mechanical system in nanoscale would be different from that in micro scale, and one should learn more from biological systems [36]. 
The time for transporting anything a distance $l$ by stirring, is about $l / v$. Whereas, for transport by diffusion, it is $l^{2} / D$, where $D$ is the diffusion coefficient in $\mathrm{cm}^{2} / \mathrm{sec}$ [28]. Namely in the micro scale,

time for transport by stirring: $\frac{l}{v}$

time for transport by diffusion: $\frac{l^{2}}{D}$,

and the ratio of these two (termed stirring number; $\S$ ) is

$$
\S \equiv \frac{\text { time for transport by stirring }}{\text { time for transport by diffusion }}=\frac{l v}{D} \approx 10^{-2}
$$

which shows the efficiency of diffusion on a small scale.

\subsubsection{Navier-Stokes}

Incompressible flow of the Navier-Stoks equation is

$$
\rho \frac{\partial \mathbf{v}}{\partial t}+\rho(\mathbf{v} \cdot \nabla) \mathbf{v}=-\nabla P+\eta \nabla^{2} v
$$

where $P$ is the pressure. Ignoring the term of inertia, and considering the large $\eta$ it can be transformed as

$$
\nabla P=\eta \nabla^{2} v
$$

It is known that the motions which are that invariant under time reversal does not induce a tow movement.

\subsubsection{Diffusion Equation}

Consider a particle that exists at $x=0$ at $t=0$. The positioning probability $(\rho(x, t))$ of $x$ follows the diffusion equation:

$$
\frac{\partial \rho}{\partial t}=D \frac{\partial^{2} \rho}{\partial x^{2}}
$$

where $D$ is a diffusion constant.

Considering the initial condition $\rho(x, 0)=\delta(x)$, and taking that the $\rho$ satisfies the following normalized condition

$$
\int_{-\infty}^{\infty} \rho(x, t) d x=1,
$$

we obtain

$$
\rho(x, t)=\frac{1}{\sqrt{4 \pi D t}} \exp \left(-\frac{x^{2}}{4 D t}\right),
$$

which obeys the Gaussian distribution. 
The mean-square displacement $\left\langle x^{2}\right\rangle$ can be derived as

$$
\left\langle x^{2}\right\rangle=\int_{-\infty}^{\infty} x^{2} \rho(x, t) d x=2 D t \propto t
$$

where $D=\frac{k_{B} T}{\zeta}, k_{B}$ is the Boltzmann constant and $\zeta$ is a friction coefficient.

In our scale, where the viscosity is negligible, using agitation for traveling is a good tactic. Whereas in the molecular scale, Brownian motion enables the speedy spacial transitions. It is in the intermediate scale $(\mu \mathrm{m})$, where those tactics lose validity because of the high viscosity and relatively small momentum.

\section{4 (C) Interaction}

The third concern is about physical interactions among components. The scalability of physical interaction mechanisms - especially electrostatic and magnetic - are well examined in [11, 1]. Here we briefly describe the basics of these two physical quantities.

\subsubsection{Electrostatic Interactions}

Given that an electric charge $q_{i}$ exists. The electric field $E$ created by this charge is

$$
\mathbf{E}=\frac{q_{i}}{4 \pi \varepsilon_{0}} \frac{\hat{\mathbf{r}}}{|\mathbf{r}|^{2}} .
$$

where $\varepsilon_{0}$ is the electric permittivity of free space.

The force $F_{j i}$ that electric charge $q_{j}$ receives is given by

$$
\begin{aligned}
\mathbf{F} & =q_{2} \mathbf{E} \\
& =\frac{q_{1} q_{2}}{4 \pi \varepsilon_{0}} \frac{\hat{\mathbf{r}}}{|\mathbf{r}|^{2}} .
\end{aligned}
$$

Therefore the decay of force over space is identical regardless of the scales.

\subsubsection{Magnetism}

We consider the magnets as dipoles with a magnetic moment $m$. The magnetic potential $\phi_{j}(r)$ at a position $r$ due to the magnetic moment $m_{j}$ is given by

$$
\phi_{j}(r)=\frac{\mu_{0}}{4 \pi} \frac{m_{j} \cdot \hat{r}}{r^{2}}
$$

where $\mu_{0}=4 \pi \times 10^{-7} \mathrm{Tm} / A$ is the permeability of free space, and $\hat{r} \equiv r /|r|$ assuming that $|r|=r$ is much larger than the size of the magnet. The magnetic flux of the dipole is then given by

$$
B_{j}=-\nabla \phi_{j}
$$


and the magnetic potential energy $U_{i j}$ acquired by a second dipole $m_{i}$ placed in the field of $m_{j}$ is given by

$$
U_{i j}=-m_{i} \cdot B_{j}
$$

Then, the force between the two dipoles is found by differentiating (14) with respect to $r$.

$$
\begin{aligned}
F_{i j} & =\left(m_{i} \cdot \nabla\right) B_{j} \\
\tau_{i j} & =m_{i} \times B_{j}
\end{aligned}
$$

We can determine the total potential energy of the system as

$$
U_{\text {total }}=\frac{1}{2} \sum_{i, j i \neq j} U_{i j} .
$$

\subsection{The Engineering Issues - Actuator Battery Connector Bottleneck}

For modular systems smaller than a few $\mathrm{cm}$, there are three fundamental problems that still await a solution. These problems relate to actuator, battery (or power in general), and connector technology. When designing systems where a high quantity of components of small size is desired, solutions for these problems are of particular relevance. First, actuation endows the parts with the ability to move and re-configure. A common solution is to use electrical servo motors. These actuators, however, are typically big and heavy. Other means of actuation have also been proposed, e.g. pneumatic actuators. Although they are lightweight, they require a source of compressed air (e.g. a compressor). The second problem is concerned with providing power to the actuator(s). A typical solution is to use batteries. Batteries, however, are problematic, because they are only able to provide power for a limited amount of time. Furthermore, their initial charge may vary which leads to heterogeneously actuated components. Another popular solution involves propagating current through the binding locations. Unfortunately, this solution has the drawback that the alignment of the connecting points has to be very precise. In addition, such components can not segregate from the main structure which prohibits this way of powering for mobile type robots. The third problem is the connection mechanism enabling the modular parts to dock to each other. Binding is crucial for reorganization and for a desired structure to hold. The most common ways of binding are magnets and mechanical latches.
A Actuator
B Battery
C Connector

There is a strong interdependency between these issues. The requirements of the connection mechanism as well as the actuator are partly determined by the weight of each component. The heavier the components are, the more force needs to be 
applied to the binding location. In addition, the actuators have to apply larger torques to displace the components. The use of more powerful components in general leads to even heavier components. Also, the power consumption increases as a result of stronger connection mechanisms and actuators. Surprisingly, small size and weight reduction of modular parts is not a good way to solve this problem, because not only does the power/weight ratio of the most common actuators decrease with a reduction in size, but also so does the strength/weight ratio of common connectors. This implies that the most common ways of actuating, powering and connecting modular robots cannot be applied to small-sized entities. It follows that novel solutions to the $A B C$ bottleneck are necessary in order to make progress in small-scale self-assembly robotics.

\section{Case Study}

In this section, we discuss how to evaluate dynamics of self-assembly based on a case study employed in our group (see [25] for more details). In the experiments, we employed Tribolon platform [23] consisting of centimeter-sized modules floating on the water surface. 12 modules were prepared and equipped with permanent magnets (a single cubic permanent magnet each, whose flux density is $1.3 \mathrm{~T}$, and size is $5 \times 5 \times 5 \mathrm{~mm}^{3}$ ). They are attached to the bottom of each module orienting the same direction such that the modules repel each other (e.g. north is always pointing up, Fig. 6). Half of the modules were, in addition to the permanent magnet, also equipped with vibration motors (termed active modules, in comparison to passive modules which doesn't feature magnets) such that they can vibrate receiving power from a ceiling via pantograph. The vibrating modules are equipped with a flat coreless vibration motor (T.P.C DC MOTOR FM34F, $12000 \sim 14000 \mathrm{rpm}(2.5-3.5$ Volts $))$ on the top of the base plate to induce selfpropulsive motion. When an electrical potential was applied to the ceiling plate (Fig.6b), current flowed through the pantograph to the vibration motor was applied to the ceiling plate, current returning to ground via electrodes immersed in the conductive water. Due to this setup, all modules receive the same constant power and they are be lightweight ( $2.8 \mathrm{~g}$ each), which would not be the case if batteries were used.

We conducted 15 trials for the statistical analysis. In Fig.7, we show a representative result in time sequence of the obtained segregation behavior. The initial starting condition was set as depicted in Fig.7 (00:00), in which all the modules were symmetrically aligned in a circular form alternately, such that the passive and the vibrating modules have equal chances in the segregation process. The duration time for the experiment was set to 90 seconds.

In order to perform the analysis, the trajectories (positions) of all the modules were tracked using the open source tracking software Tracker Video Analysis and Modeling Tool [9]. Our observation is that the red active modules tend to assemble together and go apart from the blue passive modules, such that two different 

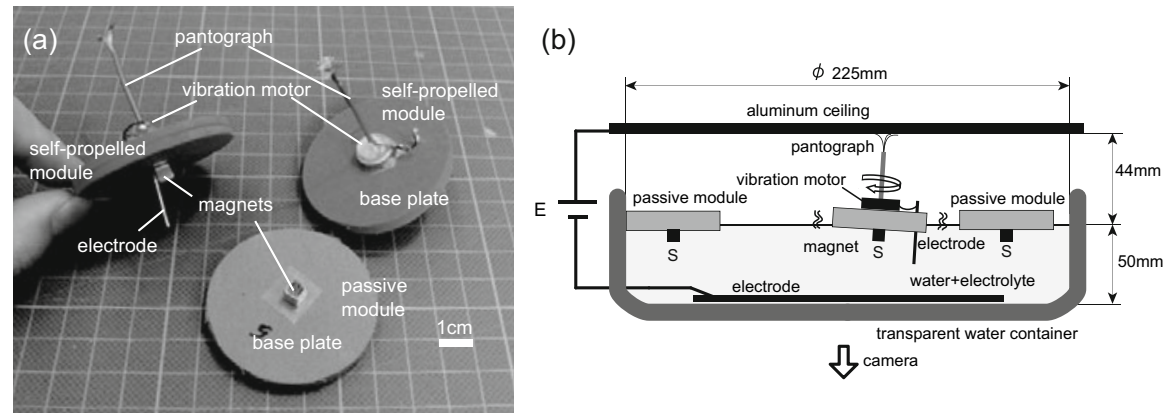

Fig. 6 (a) Self-propelled and passive modules. Each module weighs $2.8 \mathrm{~g}$ and has a footprint of $12.25 \mathrm{~cm}^{2}$.(b) Illustration of the experimental environment with three modules.

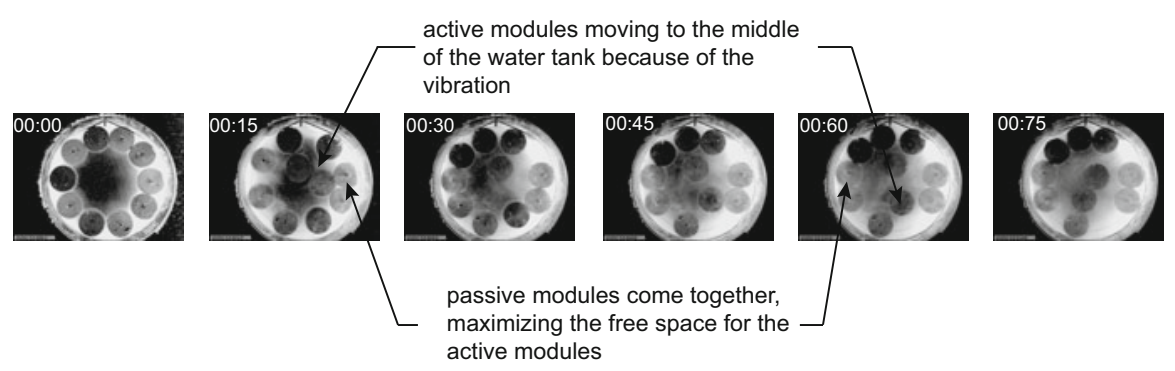

Fig. 7 The experimental results in time sequence. The frames are captured every 15 seconds

modules clusters can be spatially distinguished; the first cluster contains only the active modules and the second cluster the passive modules (Fig.7(00:75)).

\subsection{Magnetic Potential Energy and Centroid Distance}

We defined the magnetic potential energy of the system as $U_{\text {total }}^{\prime} \equiv U_{\text {total }} /\left(\frac{\mu_{0}}{4 \pi} m^{2}\right)$ by normalizing the energy defined in Eq. 17, and show the obtained result in Fig.8](a) as function of time. The error bars represent the standard deviation of uncertainty within the fifteen experimental trials. Due to the characteristics of the system, namely non-equilibrium system, the magnetic potential energy keeps decreasing. Suppose we have all passive modules, the system is supposed to reach to the state where modules are equally distributed and fixed.

The centroid $(X, Y)=\left(\frac{1}{N} \sum_{i=1}^{N}\left(x_{i}\right), \frac{1}{N} \sum_{i=1}^{N}\left(y_{i}\right)\right)$ of a group (or cluster) of modules is the center of mass of the modules, where $N$ is the number of modules in the modules group, $x_{i}$ and $y_{i}$ are the positions of the $i$-th component of the considered group, respectively. We calculated the time evolution of the difference between the two modules groups (the passive modules on one side and the active modules on the second side and depicted in Fig. 8(b). As depicted in Fig.8(b), there is an increase in the distance between the centroids of the passive and the vibrating modules. This 
(a)

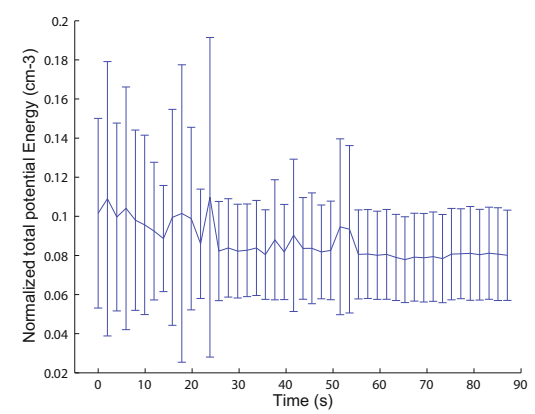

(b)

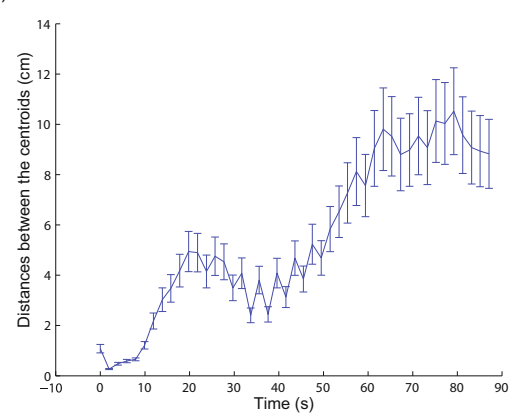

Fig. 8 (a) total energy of the system. (b) time evolution of the distance to between the center of mass of the two clusters $(N=15)$.

corresponds to the formation of two clusters of modules with a final mean distance between the two clusters of approximately 10 centimeters. Given that the diameter of the arena (or tank as you wish) is $22.5 \mathrm{~cm}$, this corresponds to the $50 \%$ of the whole area.

\subsubsection{Entropy for Transient State (Hierarchic Social Entropy)}

The definition of entropy differs in scientific fields, depending on to what one applies. Thermodynamics entropy (to heat), statistical mechanics entropy (to object), and information entropy (to event) are probably the three best known entropies in science. In self-assembly, systems that cannot presume some specific physical amounts, such as quantity of heat, employ information entropy for the measurement of their "randomness".

Balch proposed a novel definition of entropy (position order) that can be applied for the measurement of multi-components distributions (or quantitative metric of diversity) [2]. He uses $H$ from Shannon's theory

$$
H(h)=-\sum_{i=1}^{N} p_{i}(h) \log _{2}\left(p_{i}(h)\right)
$$

where $p_{i}$ is the number of modules in the $i-t h$ cluster $(i \in N)$ divided by the total number of modules. A component belongs to a cluster if the distance is within the length of $h\left(\left\|\mathbf{r}_{\mathbf{i}}-\mathbf{r}_{\mathbf{j}}\right\|<h ; \mathbf{r}_{\mathbf{i}}\right.$ is the position of the $i$-th component). He then integrates $H(h)$ over all possible $h$, and defines it as entropy, namely:

$$
S=\int_{0}^{\infty} H(h) d h .
$$

The definition describes the randomness of modules well. Note that in this definition, the entropy may decreases over time. In physics, an entropic force acting in a system is a macroscopic force whose properties are primarily determined not by the 
character of a particular underlying microscopic force (such as electromagnetism), but by the whole system's statistical tendency to increase its entropy. We examined the entropy of the system as derived as in Eq.19. Fig.9 9 shows the time evolution of the entropy of the system.

As we can observe, the entropy of the system is decreasing as time progresses, which represents the convergence of the system to more ordered configurations. This corresponds to the cluster formation described of the previous section.

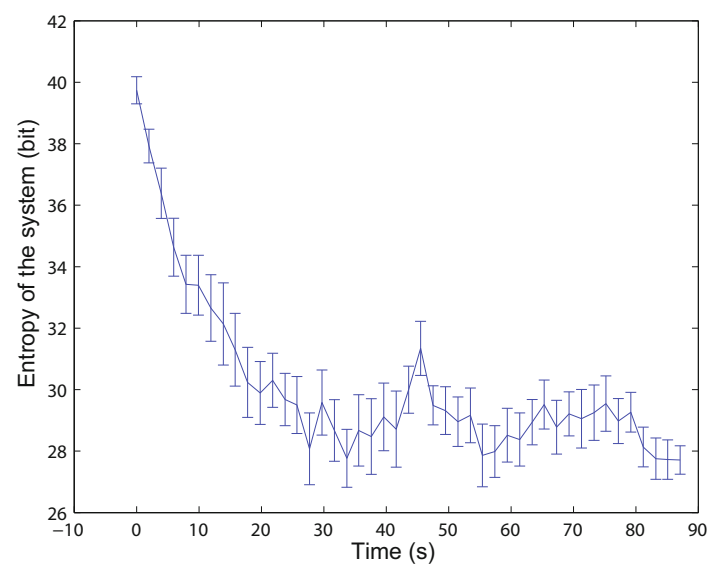

Fig. 9 Transition of entropy.

\subsubsection{Transfer Entropy}

To see the simple informational structure, we used a measure that aims at extracting directed flow (transfer of information) between time series of both active and pssive modules, called transfer entropy [29]. Given two arbitrary time series $x_{t}$ and $y_{t}$, transfer entropy essentially quantifies the deviation from the generalized Markov process: $p\left(x_{t+\tau} \mid x_{t}\right) \approx p\left(x_{t+\tau} \mid x_{t}, y_{t}\right)$, where $p$ denotes the transition probability. If the deviation from a generalized Markov process is small, then the state of $Y$ can be assumed to have little relevance on the transition probabilities of system $X$. If the deviation is large, however, then the assumption of a Markov process is not valid. The incorrectness of the assumption can be expressed as follows:

$$
T E(Y \rightarrow X)=\sum_{x_{t+\tau}} \sum_{x_{t}} \sum_{y_{t}} p\left(x_{t+\tau}, x_{t}, y_{t}\right) \log \frac{p\left(x_{t+\tau} \mid x_{t}, y_{t}\right)}{p\left(x_{t+\tau} \mid x_{t}\right)}
$$

where the sums are over all amplitude states, and the index $T E(Y \rightarrow X)$ indicates the influence of $Y$ on $X$. The transfer entropy is explicitly nonsymmetric under the exchange of $X$ and $Y$, and can thus be used to detect the directed exchange of information between two systems. 
Here, $Y$ is a time series of $d_{t}^{i}$ of a module $i$ and $X$ is a time series of $d_{t}^{j}$ of a module $j$. The base of $\log$ is set to $e$ and the parameter $\tau$ is set to 1. By using transfer entropy, we aim to evaluate causal relations between all the pairs of modules.

By using the data, we simply calculated a distance of movement in each time step for each modules $\left(d_{t}^{i}\right)$ as follows.

$$
d_{t}^{i}=\sqrt{\left(x_{t+1}^{i}-x_{t}^{i}\right)^{2}+\left(y_{t+1}^{i}-y_{t}^{i}\right)^{2}}
$$

We discretized the value of $d_{t}^{i}$ ranging from 0.0 to 12.0 into $10,20,50$, and 100 bins and all the 15 trials from $0 \leq$ timesteps $\leq 44$ were used and averaged on trials. Since the number of samples is limited, we varied the bin size and saw the relevance.

We show the results in Fig 10 and 11 in which the module number 1 to 6 are passive modules, while 7 to 12 are active modules. As can be seen from the results of Bin=10 (Fig 10 a)), 20 (Fig 10 b)), and 50 (Fig 11 a)), the values of TE (active $\rightarrow$ active) are high. Also, the values of $T E$ (active $\rightarrow$ passive), $T E$ (passive $\rightarrow$ active) are relatively high, and the values of $T E$ (passive $\rightarrow$ passive) are low. These results simply suggest that active elements influence the transitions of active elements and passive elements. On the other hand, passive elements only influence the transitions of active elements. And the values of $T E(Y \rightarrow X)$ are simply the degree of its influence. These results fit well to our natural observations of the system. On the result of Bin=100 (Fig 11 b)), since the number of samples (observed time steps) are relatively small for the size of the state space, it cannot structure the relevant probability density.

(a) Bin=10

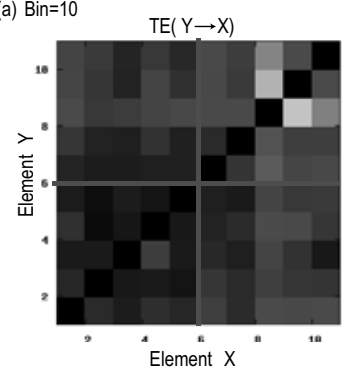

(b) $\mathrm{Bin}=20$

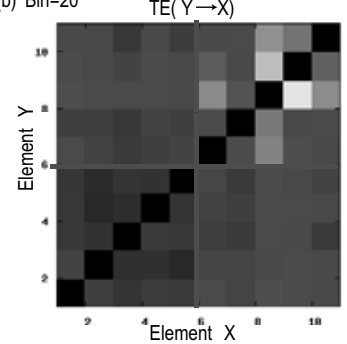

SD

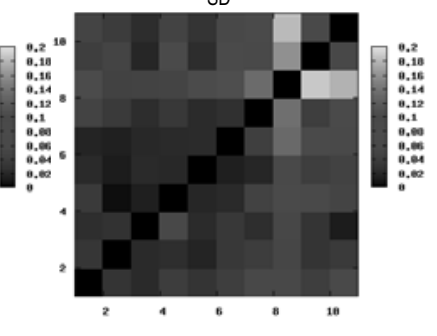

SD

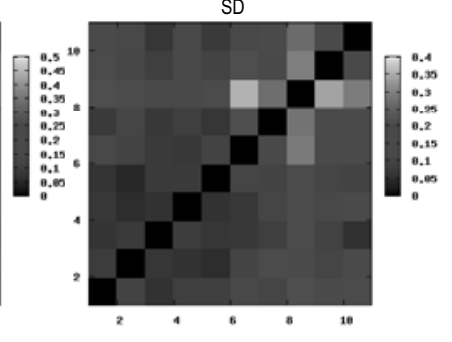

Fig. $10 T E(Y \rightarrow X)$ and standard deviations (SD). (a) bin=10, (b) bin=20. 

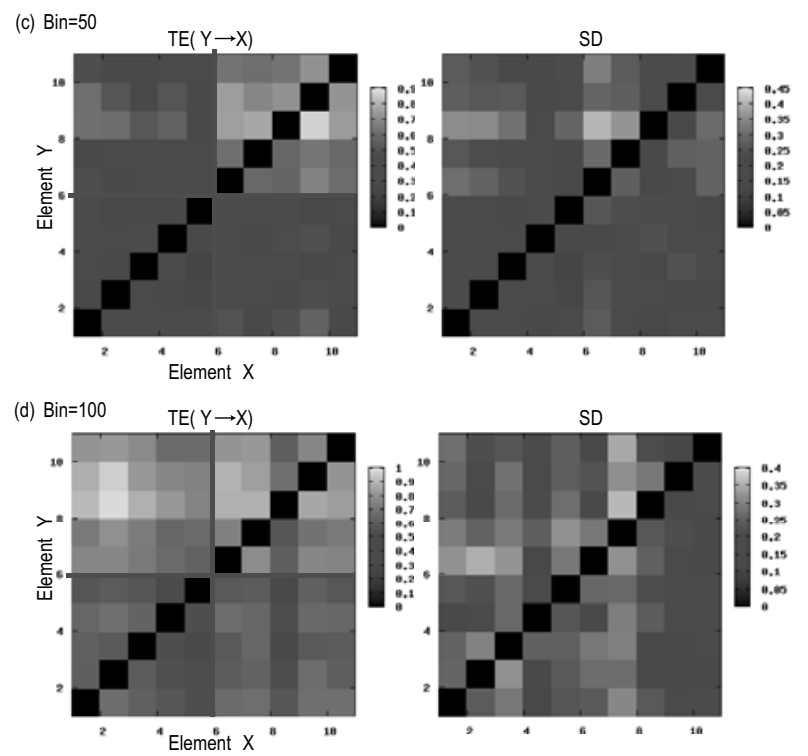

Fig. $11 T E(Y \rightarrow X)$ and standard deviations (SD). (c) bin=50, (d) bin=100.

In this system, transfer entropy tends to show $T E$ (active $\rightarrow$ active) $>$ $T E($ active $\rightarrow$ passive $), T E($ passive $\rightarrow$ active $)>T E($ passive $\rightarrow$ passive $)$. This result naturally fits to our simple observation of the trajectories.

In our results, the values of standard deviations (SD) for transfer entropy were large. This is caused by the small number of data samples (time steps). Considering the experimental setting, reasonable extensions of the experimental time steps are recommended. Additionally, to detect the causal relation, we can use other measures, such as granger causality, mutual information, symbolic transfer entropy [32], etc., according to what we would like to see. Especially, by using symbolic transfer entropy [32], we can avoid the difficulty to set the bin size. But in this case, extensions of timesteps are inevitable.

Although we calculated the information transfer between modules, it is also possible to measure causal relations between the global behavior and the elements [33]. By doing this, we can detect how each element affect the global behavior, and how the global behavior regulates each element quantitatively.

\section{Conclusions}

In this paper, we systematically studied various problems on self-assembling systems. Starting from pointing to some fundamental concerns of self-assembly, we 
categorized them into three basic issues, namely on assembly, dynamics, and interactions. We examined quantification methods utilizing a case study in which modules showed segregation behavior in a distributed way. We further investigated the possible style of description of entropy as well as free energy that can govern macroscopic self-assembly systems. We believe the clarification of basic problems in self-assembly and proper assignment of an approaching method will offer new opportunities to deepen the theoretical understanding of the phenomenon, and will lead to the realization of efficient self-assembly systems.

\section{Acknowledgment}

This research was supported by the Swiss National Science Foundation project \#200020118117 .

\section{References}

1. Abbott, J.J., Nagy, Z., Beyeler, F., Nelson, B.J.: Robotics in the small. IEEE Robotics \& Automation Magazine 14, 92-103 (2007)

2. Balch, T.: Hierarchic social entropy: An information theoretic measure of robot group diversity. Autonomous Robots 8, 209-237 (2000)

3. Boncheva, M., Andreev, S.A., Mahadevan, L., Winkleman, A., Reichman, D.R., Prentiss, M.G., Whitesides, S., Whitesides, G.: Magnetic self-assembly of three-dimensional surfaces from planar sheets. PNAS 102, 3924-3929 (2005)

4. Boncheva, M., Ferrigno, R., Bruzewicz, D.A., Whitesides, G.M.: Plasticity in selfassembly: Templating generates functionally different circuits from a single precursor. Angew. Chem. Int. Ed. 42, 3368-3371 (2003)

5. Boncheva, M., Gracias, D.H., Jacobs, H.O., Whitesides, G.M.: Biomimetic selfassembly of a functional asymmetrical electronic device. PNAS 99, 4937-4940 (2002)

6. Bowden, N., Terfort, A., Carbeck, J., Whitesides, G.M.: Self-assembly of mesoscale objects into ordered two-dimensional arrays. Science 276, 233-235 (1997)

7. Bowden, N., Weck, M., Choi, I.S., Whitesides, G.M.: Molecule-mimetic chemistry and mesoscale self-assembly. Acc. Chem. Res. 34, 231-238 (2001)

8. Breivik, J.: Self-oranization of template-replicating plolymers and the spontaneous rise of genetic information. Entropy 3, 273-279 (2001)

9. Brown, D.: Tracker video analysis and modeling tool (2009), http: / / www. cabrillo.edu/ dbrown/tracker/

10. Cohn, M.B., Kim, C.-J.: Self-assembling electrical networks: An application of micromachining technology. In: International Conference on Solid-State Sensors and Actuators, pp. 490-493 (1991)

11. Cugat, O., Delamare, J., Reyne, G.: Magnetic micro-actuators and systems (MAGMAS). IEEE Trans. Magnetics 39(5), 3607-3612 (2003)

12. Demaine, E.D., Hohenberger, S., Liben-Nowell, D.: Tetris is hard, even to approximate. Technical report, Cornell University Library (2002), arXiv.org 
13. Gracias, D.H., Tien, J., Breen, T.L., Hsu, C., Whitesides, G.M.: Forming electrical networks in three dimensions by self-assembly. Science 289, 1170-1172 (2000)

14. Griffith, S., Goldwater, D., Jacobson, J.: Robotics: Self-replication from random parts. Nature 437, 636 (2005)

15. Grzybowski, B.A., Radkowski, M., Campbell, C.J., Lee, J.N., Whitesides, G.M.: Selfassembling fluidic machines. App. phys. lett. 84, 1798-1800 (2004)

16. Grzybowski, B.A., Stone, H.A., Whitesides, G.M.: Dynamic self-assembly of magnetized, millimetre-sized objects rotating at a liquid-air interface. Nature 405, 1033 (2000)

17. Grzybowski, B.A., Winkleman, A., Wiles, J.A., Brumer, Y., Whitesides, G.M.: Electrostatic self-assembly of macroscopic crystals using contact electrification. Nature 2, 241-245 (2003)

18. Hosokawa, K., Shimoyama, I., Miura, H.: Dynamics of self-assembling systems: Analogy with chemical kinetics. Artificial Life 1(4), 413-427 (1994)

19. Ishiguro, A., Shimizu, M., Kawakatsu, T.: A modular robot that exhibits amoebic locomotion. Rob. Aut. Sys. 54, 641-650 (2006)

20. Klavins, E.: Programmable self-assembly. IEEE Cont. Sys. Mag. 27, 43-56 (2007)

21. Wilson, M., Melhuish, C., Sendova-Franks, A.: Multi-object segregation: ant-like brood sorting using minimalism robots. In: Proc. Seventh International Conf. on the Simulation of Adaptive Behaviour, Edinburgh, UK, pp. 369-370 (2002)

22. Mao, C., Thalladi, V.R., Wolfe, D.B., Whitesides, S., Whitesides, G.M.: Dissections: Self-assembled aggregates that spontaneously reconfigure their structures when their environment changes. J. Am. Chem. Soc 124(49), 14508-14509 (2002)

23. Miyashita, S., Kessler, M., Lungarella, M.: How morphology affects self-assembly in a stochastic modular robot. In: IEEE International Conference on Robotics and Automation (2008)

24. Motokawa, T.: Time of an elephant, time of a mouse. In: CHUO-KORON-SHINSHA, INC. (1992)

25. Ngouabeu, A.M.T., Miyashita, S., Füchslin, R.M., Nakajima, K., Göldi, M., Pfeifer, R.: Self-organized segregation effect on water based self-assembling robots. In: Artificial Life 12, Odense, Denmark (2010)

26. Pelesko, J.A.: SELF ASSEMBLY. Chapman \& Hall/CRC, Boca Raton (2007)

27. Penrose, L.S.: Self-reproducing. Sci. Amer. 200(6), 105-114 (1959)

28. Purcell, E.M.: Life at low reynolds number. Amer. J. Phys. 45, 3-11 (1977)

29. Schreiber, T.: Measuring information transfer. Physical Review Letters 85, 461-464 (2000)

30. Wilson, M., Melhuish, C., Sendova-Franks, A.B., Scholes, S.R., Franks, N.R., Melhuish, C.: Brood sorting by ants: Two phases and differential diffusion. Animal Behaviour 68, 1095-1106 (2004)

31. Stambaugh, J., Lathrop, D.P., Ott, E., Losert, W.: Pattern formation in a monolayer of magnetic spheres. Pysical Leview E. 68, 026207-1-026207-5 (2003)

32. Staniek, M., Lehnertz, K.: Symbolic transfer entropy. Physical Review Letters 100, 158101-158101 (2008)

33. Sumioka, H., Nakajima, K., Lungarella, M., Pfeifer, R.: Complexity detection based on bidirectional information flow (submitted)

34. Tsutsumi, D., Murata, S.: Multistate part for mesoscale self-assembly. In: SICE Annual Conference (2007) 
35. White, P., Kopanski, K., Lipson, H.: Stochastic self-reconfigurable cellular robotics. In: Proc. Int. Conf. on Robotics and Automation, vol. 3, pp. 2888-2893 (2004)

36. Whitesides, G.M.: The 'right' size in nanobiotechnology. Nature 21(10), 1161-1165 (2003)

37. Whitesides, G.M., Grzybowski, B.: Self-assembly at all scales. Science 295, 2418-2421 (2002)

38. Wolfe, D.B., Snead, A., Mao, C., Bowden, N.B., Whitesides, G.M.: Mesoscale selfassembly: Capillary interactions when positive and negitive menisci have similar amplitudes. Langmuir 19, 2206-2214 (2003)

39. Yamaki, M., Higo, J., Nagayama, K.: Size-dependent separation of colloidal particles in two-dimensional convective self-assembly. American Chemical Society 11, 2975-2978 (1995) 